\title{
Metodología para el desarrollo del perfil del asesor pedagógico de educación básica ${ }^{1}$
}

\section{Methodology for developing the profile of the pedagogical counselor in basic education}

\begin{abstract}
Graciela Cordero
Doctora en Filosofía y Ciencias de la Educación, Universidad de Barcelona. Licenciada en Pedagogía, Universidad Nacional Autónoma de México. Maestra en Educación, Universidad de Harvard. Investigadora, Instituto de Investigación y Desarrollo Educativo, Universidad Autónoma de Baja California (UABC). Investigador Nivel II - Sistema Nacional de Investigadores (SNI). Miembro del Consejo Técnico de evaluación de docentes y directivos, Instituto Nacional para la Evaluación de la Educación, México.
\end{abstract}

María del Ángel Vázquez

Licenciada en Ciencias de la Educación y Maestra en Ciencias Educativas, Universidad Autónoma de Baja California (UABC). Analista, Coordinación de Formación Continua de Maestros en Servicio, UABC. Investigadora en asesoría técnico-pedagógica y en los perfiles de desempeño de los actores educativos.

Edna Luna Serrano

Doctora en Educación, Universidad Autónoma de Sinaloa, México. Master en Modificación de Conducta y Licenciada en Psicología, Universidad Nacional Autónoma de México. Investigadora del Instituto de Investigación y Desarrollo Educativo, Universidad Autónoma de Baja California. Investigadora en evaluación educativa, evaluación de la docencia y la formación profesional. Investigador Nivel II - Sistema Nacional de Investigadores (SNI). Coordinadora, Red de Investigadores sobre Evaluación de la docencia. Editora científica, Revista Electrónica de Investigación Educativa.

Fecha de recibido: 08/04/2015

Fecha de aceptado: 28/05/2015

\section{Resumen}

El objetivo de este artículo es mostrar la metodología seguida en la construcción de un perfil referencial para determinar las funciones del asesor técnico-pedagógico en una entidad federativa de México. Este proyecto se contextualiza en el marco de la Reforma Educativa que actualmente se implementa en este país. El proyecto se desarrolló en dos etapas de trabajo. En la primera etapa se definió el encuadre teórico para el diseño del perfil referencial y se hizo un análisis de contenido de la normativa vigente mexicana. El resultado de este análisis fue una primera propuesta de los tres niveles más genéricos del perfil: dominios, subdominios y competencias de la función de asesoría pedagógica. En la segunda etapa se conformaron tres comités de trabajo integrados por profesores, asesores, directores y supervisores de todos los niveles educativos y de toda la entidad para el desarrollo del perfil. El comité técnico llevó a cabo la revisión de la propuesta inicial y elaboró los parámetros para cada una de las competencias. El comité académico revisó la aportación del comité anterior y elaboró los indicadores de cada subdominio de la función asesora. Más adelante, el comité de validación revisó y calificó el trabajo realizado por los comités anteriores a partir de los criterios de congruencia, relevancia, claridad y suficiencia. Los resultados muestran un alto grado de acuerdo con el trabajo 
desarrollado por los comités anteriores. Finalmente se presenta un ejemplo del perfil validado, así como recomendaciones generales para el uso del perfil en el marco de la reforma educativa mexicana.

Palabras clave: Metodología, educación básica, regulaciones federales, estándares estatales, asesores pedagógicos, desempeño de asesores, análisis de puestos, desarrollo profesional.

\begin{abstract}
The aim of the present paper is to show the methodology used in the construction of the profile and role of an educational counselor in one of Mexico's States. This project is framed within the educational reform being currently implemented in this country. The project was developed in two stages. The first stage was devoted to the definition of the theoretical framing for designing the reference profile. A content analysis of the national regulations concerning the figure of the educational counselor was carried out. The result of such analysis was an initial proposal for the three generic levels of the profile: domains, sub domains and qualifications pertaining to the figure of educational counselor. In the second stage, three committees composed of teachers, counselors, principals, and supervisors at all levels of education and from the entire educational entity were organized with the purpose of developing said profile. The technical committee revised the original proposal and developed the parameters for each of the competencies. The academic committee reviewed the contribution of the previous committee and developed indicators for each sub domain of the counselor's role. Later, the validation committee reviewed the work carried out by both previous committees based on the criteria of consistency, relevance, clarity, and sufficiency. The results show a high degree of agreement with the work undertaken by previous committees. Finally an example of the validated profile is submitted together with general recommendations for the use of said profile within the context of the Mexican educational reform.
\end{abstract}

\title{
Introducción
}

Este artículo presenta el proceso de elaboración del perfil referencial propuesto para el asesor técnico-pedagógico (ATP) de las escuelas de educación básica en Baja California, entidad federativa del norte de México.

México vive actualmente un proceso de Reforma Educativa. Este proceso de reforma inició con la modificación del artículo tercero de la constitución en febrero de 2013 y las subsecuentes reformas a la Ley General de Educación (DOF, 2013c), así como la creación de la Ley del Instituto Nacional para la Evaluación de la Educación (DOF, 2013b) y de la Ley General del Servicio Profesional Docente (DOF, 2013d) en setiembre de 2013. Esta reforma, que ha sido calificada de "estructural" (Ornelas, 2013 y Zebadúa, 2013), recupera la autoridad del estado mexicano en la regulación de la carrera docente, competencia que había sido compartida con los sindicatos de trabajadores de la educación.

En particular, la Ley General del Servicio Profesional Docente (LGSPD) (Diario Oficial de la Federación [DOF], 2013d) es el instrumento jurídico que regula la vida profesional del profesorado: ingreso, promoción, permanencia y reconocimiento. Esta ley pretende asegurar una educación de calidad a partir de garantizar que los actores educativos reúnan las cualidades personales y competencias profesionales que demanda el servicio, por lo que todos los profesionales de la educación deben ingresar al servicio educativo o ser promocionados a otros cargos por medio de un concurso de oposición y presentar evaluaciones periódicas que den evidencia de la calidad de su desempeño. 
Para definir las bases sobre las que se implementarán los concursos de oposición para la selección de los mejores maestros o la promoción a otros cargos como dirección, supervisión y asesor pedagógico -así como la evaluación obligatoria para el reconocimiento y la permanencia en el servicio-, la LGSPD establece que deben desarrollarse perfiles referenciales de la función. En la ley, dichos perfiles se definen como el conjunto de características, cualidades o aptitudes que deberá tener el aspirante a desempeñar un puesto o función y que se describen en parámetros e indicadores (DOF, 2013d).

De acuerdo a la distribución de competencias establecida en la LGSPD, las entidades federativas deben proponer a la Secretaría de Educación Pública federal (SEP) sus propuestas de perfiles, parámetros e indicadores para el ingreso, promoción, permanencia $y$, en su caso, de reconocimiento de todos los actores educativos. Cada entidad define el procedimiento para elaborar sus propuestas. La SEP reúne las propuestas de las 32 entidades federativas y las integra en una única versión nacional. Por su parte, el Instituto Nacional para la Evaluación de la Educación es responsable del cuidado de la calidad técnica de los perfiles que emite la SEP (DOF, 2013b). Si bien el primer ejercicio de elaboración estatal y nacional de perfiles, parámetros e indicadores de docentes (SEP, 2014) se hizo a lo largo de 2014, no se conocen documentos que describan la metodología desarrollada ni en la entidades federativas, ni a nivel nacional.

En nuestro caso, la propuesta se desarrolló en la Universidad Autónoma de Baja California en acuerdo con la Secretaría de Educación y Bienestar Social de Baja California (SEBS). El objetivo del proyecto fue construir el perfil referencial de competencias del personal docente con funciones de Asesor Técnico-pedagógico (en adelante ATP) para el desarrollo y mejora continua de las escuelas de educación básica de Baja California a partir de la perspectiva de los actores educativos (tanto en calidad de usuarios -profesores y directivos-, como de agentes de apoyo externos -asesores, supervisores, inspectores-). Este artículo es un reporte parcial del proyecto y tiene el objetivo de describir la metodología de trabajo desarrollada a lo largo del proyecto, en tanto que se considera que fue innovadora en nuestro contexto educativo.

La propuesta se hizo siguiendo las recomendaciones de la Oficina Regional de Educación de la Organización de las Naciones Unidas para la Educación, la Ciencia y la Cultura para América Latina y el Caribe (Orealc/Unesco, 2013) en el sentido de diseñar estrategias para la construcción y revisión de perfiles profesionales en los que participen los actores involucrados en los procesos educativos. Este organismo señala que para desarrollar sistemas válidos de formación y evaluación del desempeño se requiere definir criterios objetivos, transparentes, basados en estándares consensuados y validados por los propios profesionales de la educación, considerando el contexto escolar.

El artículo está estructurado en cuatro secciones. La primera, de carácter introductorio, presenta una breve caracterización de los asesores en el sistema educativo mexicano y el encuadre teórico desde el cual se desarrolló el proyecto. En una segunda sección se incluye la metodología del estudio. En tercer término se muestra el perfil referencial elaborado como resultado del estudio y se incluye un ejemplo del perfil del asesor para la dimensión de asesoría y acompañamiento. Finalmente se presentan algunas conclusiones sobre el uso del perfil en el contexto de la reforma educativa mexicana. 


\section{La figura del asesor técnico-pedagógico en el sistema educativo mexicano}

Previo a la Reforma Educativa, en el sistema educativo mexicano el asesor era un profesor comisionado o adscrito a esta función a nivel escuela, zona, sector o programa, que se desempeñaba en ámbitos de trabajo comúnmente definidos por los requerimientos particulares de sus superiores. Sus funciones estaban muy relacionadas a las actividades de tipo administrativo. La figura del asesor se caracterizó, entre otras cosas, por la indefinición del perfil y funciones, por la falta de criterios para evaluar su ingreso y para dar seguimiento a su desempeño y por la ausencia de programas específicos para su formación (Bonilla, 2006; Calvo, 2007; Meza, Vázquez, y Cordero, 2008; Sánchez, Cordero y Bocanegra, 2009; Tapia, 2008; Vázquez, Meza, Cordero y Patiño, 2012; Vázquez, Meza y Cordero, 2012; SEP 2005; SEP, 2012).

De acuerdo con Arnaut (2006), en México la formación de los primeros cuadros de apoyo técnico-pedagógico a la escuela se dio a finales de 1970. A partir de la reforma educativa de 1992, ante la necesidad de consolidar los equipos de trabajo de las supervisiones escolares para promover la implementación de los nuevos planes y programas de estudio y los programas de apoyo a la escuela, se requirió un mayor número de profesores que desempeñaran esta función. De entonces a la fecha, la figura del asesor ha crecido en forma no regulada y sin una política clara que definiera el sentido pedagógico de su función. En el marco de la Reforma Educativa vigente en nuestro país, la LGSPD reconoce por primera vez la figura del asesor en el sistema educativo mexicano. El asesor técnico-pedagógico se define como:

Docente que en la Educación Básica y Media Superior cumple con los requisitos establecidos en la presente Ley y tiene la responsabilidad de brindar a otros docentes la asesoría señalada y constituirse en un agente de mejora de la calidad de la educación para las escuelas a partir de las funciones de naturaleza técnico pedagógica que la Autoridad Educativa o el Organismo Descentralizado le asigna (DOF, 2013d, p. 4).

En el marco de la ley, ser asesor se considera un tipo de promoción en el servicio. El artículo 41 de la LGSPD establece la posibilidad de ingresar a esta función por concurso de oposición y obtener un nombramiento definitivo en esta categoría. Asimismo, esta ley establece que este personal por ningún motivo podrá desempeñar funciones del ámbito administrativo.

\subsection{Encuadre para la construcción del perfil del ATP}

Durante los primeros años de este siglo los sistemas educativos han venido introduciendo reformas para mejorar la calidad de la enseñanza basadas en el establecimiento de una política integral para seleccionar, preparar, desarrollar y evaluar a los docentes. Con este fin se definen y establecen perfiles y estándares que precisan los conocimientos, habilidades y valores relacionados con la buena enseñanza (Celis, 2012). Dichos perfiles se integran por componentes que representan niveles de desagregación de sus funciones. A partir de estos referentes se articulan estrategias para fortalecer la carrera docente, tales como:

- Establecer un marco de actuación del profesional, puesto que define sus competencias y delimita sus funciones.

- Diseñar programas de formación para los profesionales.

- Desarrollar estrategias e instrumentos para la evaluación del ingreso, la permanencia y la promoción en la función.

- Realizar procesos de evaluación formativa para reflexionar sobre la práctica e identificar ámbitos de mejora (Barrera y Myers, 2011; Ingvarson y Kleinhenz, 2006; Vaillant, 2004). 
En México, el primer proyecto nacional para el establecimiento de perfiles profesionales en educación se llevó a cabo en 2010 (SEP, 2010). En este proyecto se elaboraron estándares de desempeño para docentes y directores. Dichos estándares estaban organizados en cinco niveles: categorías, referentes, estándares, niveles de desempeño y pautas para observar. Este proyecto no llegó a ser discutido a nivel nacional ni integrado a la legislación mexicana.

A partir de la LGSPD, se establece por ley contar con un perfil que defina el desempeño de docentes, directivos, supervisores y asesores técnico-pedagógicos. Como se mencionó anteriormente, el perfil es requerido tanto para el ingreso como para la promoción y la permanencia. La LGSPD identifica solo dos componentes de dicho perfil: parámetros2 e indicadores. La ley define parámetro como "el valor de referencia que permite medir avances y resultados alcanzados en el cumplimiento de objetivos, metas y demás características del ejercicio de una función o actividad" (DOF, 2013d, p. 4). Asimismo, define indicador como "el instrumento utilizado para determinar, por medio de unidades de medida, el grado de cumplimiento de una característica, cualidad, conocimiento, capacidad, objetivo o meta, empleado para valorar factores que se desean medir" (DOF, 2013d, p. 3).

A fin de la identificación de la estructura más adecuada para el perfil a construir, se llevó a cabo una revisión de perfiles y marcos de buen desempeño de varios países (Danielson, 2013; Chile, Gobierno de Chile, 2011; Perú, Ministerio de Educación de Perú, 2012). Se observó que no hay un acuerdo en los conceptos utilizados para denominar cada nivel, así como tampoco existe acuerdo en los niveles de desagregación de los perfiles o marcos (Vázquez, Cordero y Leyva, 2014). Se revisó también la terminología utilizada por la propia SEP en la LGSPD (DOF, 2013d). A partir de esto, se optó por estructurar el perfil en cinco niveles de descripción, los cuales van desde una visión general del desempeño del profesional a una más específica, como se muestra en el gráfico 1.

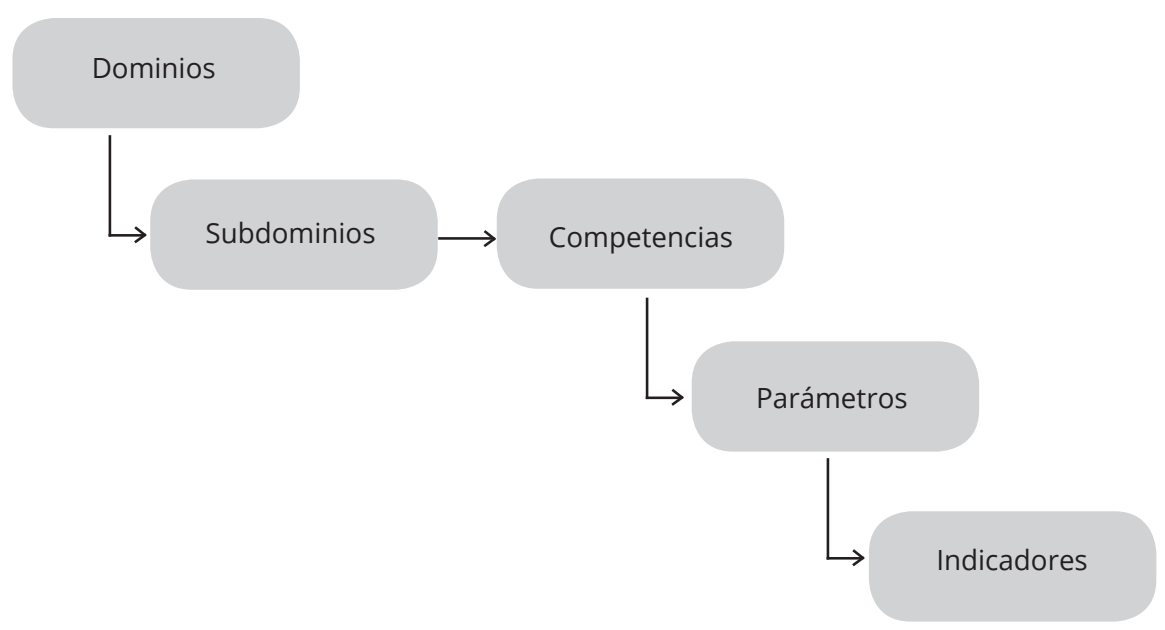

\section{Gráfico 1. Niveles de descripción del perfil seleccionados}


El primer nivel son los dominios. De acuerdo con Stronge y Tucker (2003) un dominio provee un marco general para describir el ámbito de desempeño de un profesional. Estos representan un marco general para describir la función; son las categorías más amplias en las cuales se clasifican las competencias del perfil. El segundo nivel son los subdominios que integran cada dominio. En un tercer nivel se encuentran las competencias. Existen múltiples definiciones de competencias. En este caso se entiende por competencia al conjunto de conocimientos, procedimientos y actitudes combinados, coordinados e integrados que permiten desempeñar el rol de profesional de manera eficiente (Cano, 2012). En el cuarto nivel se incluyen los parámetros, que representan los deberes básicos para cumplir con éxito las expectativas de la función (Stronge y Tucker, 2003). Especifican lo que necesitan saber y hacer los profesionales para su buen desempeño (Ingvarson y Kleinhenz, 2006), y se expresan a manera de conocimientos, habilidades y actitudes. El quinto y último nivel son los indicadores, el nivel más específico del perfil. Son ejemplos observables que caracterizan el desempeño exitoso de los parámetros o estándares (Stronge y Tucker, 2003). Se consideró que esta estructura permitía la interlocución con los diferentes actores y con las autoridades educativas federal y estatal ya que incorporaba los conceptos más utilizados en la literatura así como la terminología propia de la legislación mexicana.

\section{Método de trabajo}

El proyecto se desarrolló en dos grandes etapas. La primera tuvo como objetivo definir el encuadre del perfil referencial y elaborar una propuesta de competencias para el personal docente con funciones de asesoría técnica pedagógica (ATP) de educación básica, a partir de un análisis de los documentos oficiales que definen la función de asesoría técnicopedagógica, a nivel nacional y estatal. En la segunda etapa se construyó el perfil referencial del ATP de educación básica de Baja California, a partir del trabajo colegiado con los actores involucrados en el proceso de asesoramiento: usuarios y agentes de apoyo externos. A continuación se describe cada una de estas etapas.

\subsection{Primera etapa del proyecto. Análisis documental de las funciones del asesor técnico-pedagógico}

Esta etapa tuvo tres objetivos. El primer objetivo fue definir la estructura del perfil a construir. Un segundo objetivo fue identificar las funciones del ATP planteadas en los documentos oficiales, nacionales y estatales que definen la asesoría técnico-pedagógica, identificar los dominios y subdominios de las funciones del ATP. El tercero fue elaborar una propuesta de competencias del ATP congruentes con las funciones que plantean los documentos oficiales, nacionales y estatales.

Con este fin se llevó a cabo un análisis de contenido de los documentos oficiales que definen la función de asesoría a nivel nacional para identificar los posibles dominios del perfil referencial para el personal docente con funciones de asesoría técnico-pedagógica. El análisis de contenido del presente estudio tomó como base el procedimiento propuesto por Elo y Kyngäs (2008). Los autores definieron tres fases generales en el desarrollo de un análisis de contenido: preparación, organización y estructuración de la propuesta base de dominios y subdominios.

\subsubsection{Procedimiento}

\section{Preparación}

En la primera fase se identificaron los documentos relacionados con la figura del asesor técnico-pedagógico publicados a nivel federal y estatal. Esta lista de documentos se validó con un asesor de escuela primaria para confirmar que se hubieran tomado en cuenta todos los documentos relacionados con el tema. 
En el ámbito federal, dos publicaciones centrales han definido la función. Por una parte, la Dirección General de Formación Continua de Maestros en Servicio de la SEP emitió las "Orientaciones generales para constituir y operar el servicio de asesoría académica a la escuela" (SEP, 2005). Por otra parte, la Dirección General de Desarrollo de la Gestión e Innovación Educativa publicó los cinco tomos de la Colección "Criterios para el fortalecimiento del Sistema Regional de Asesoría Académica a la Escuela" (SEP, 2012). Cabe señalar que ambas direcciones pertenecen a la propia SEP y que en el análisis de estos documentos se detectó que no era claro al interior de la SEP cuál de las dos instancias debía regular la figura del asesor (Cordero, Fragoza y Vázquez, 2015).

Asimismo, se realizó la revisión de documentos oficiales publicados a partir de la Reforma Educativa. Los documentos revisados fueron los siguientes:

- $\quad$ Plan Nacional de Desarrollo 2013-2018 (DOF, 2013a)

- Ley del Instituto Nacional para la Evaluación de la Educación (INEE) (DOF, 2013b)

- $\quad$ Ley General de Educación (DOF, 2013c)

- $\quad$ Ley General del Servicio Profesional Docente (DOF, 2013d).

- Reglas de Operación del Programa para el Desarrollo Profesional Docente (DOF, 2013e).

A nivel de la entidad federativa, las funciones del ATP se revisaron en el documento "Lineamientos normativos para la gestión institucional, escolar y pedagógica para el ciclo escolar 2013-2014" (SEBS, 2013).

En una segunda instancia se identificaron los referentes de la asesoría que se proponen en los documentos oficiales, es decir, los textos que describen la función del asesor y que sirven como guía para su desempeño profesional. En el análisis de los documentos oficiales se identificaron listas distintas de funciones del ATP bajo denominaciones diversas: tareas y competencias, perfil de profesionalización, funciones del asesor académico, estándares de desempeño de los asesores y lineamientos para el personal docente con funciones de ATP. En estas listas se identificaron las unidades de análisis, fueran enunciados o frases. Debido a que algunas competencias, funciones y estándares implicaban más de una actividad, se disociaron para convertir cada tarea en una unidad de análisis. En total se identificaron 90 unidades de análisis.

\section{Organización}

La segunda fase implicó la categorización de las unidades de análisis para llevar a cabo una definición inicial de los dominios. Se realizó una codificación abierta en la que se identificaron los temas de las unidades de análisis a fin de establecer los dominios y subdominios de las funciones. Se integraron las unidades que se referían a la misma actividad, aunque su redacción fuera distinta, y se construyó un solo enunciado a partir de aquellas. Las unidades de análisis se ubicaron en los dominios y subdominios.

\section{Estructuración de la propuesta base de dominios y subdominios}

La tercera fase consistió en la elaboración de una primera propuesta de dominios y subdominios, a partir de las funciones identificadas en los documentos oficiales. Este material fue el insumo básico para la segunda etapa de la investigación.

\subsection{Segunda etapa del proyecto. Construcción del perfil referencial del ATP}

Consistente con las recomendaciones de la Orealc/Unesco (2013), en esta segunda etapa se diseñó un procedimiento para la integración de tres grupos de trabajo: comité técnico, comité académico y comité de validación, cada uno con una función particular. La etapa tuvo los siguientes objetivos específicos: 
1. Revisar y analizar la propuesta de dominios y subdominios identificados en la etapa anterior.

2. Definir las competencias del ATP de educación básica de Baja California.

3. Definir los parámetros del ATP de educación básica Baja California congruentes con las competencias planteadas.

4. Establecer los indicadores del desempeño de los ATP.

5. Validar los parámetros e indicadores de desempeño del ATP de educación básica del Estado de Baja California

\subsubsection{Procedimiento}

\section{Selección de los participantes}

Los profesionales de la educación que participaron en el proceso de elaboración del perfil del ATP se seleccionaron de acuerdo con los siguientes criterios:

- Más de cinco años de experiencia laborando en alguno de los niveles o tipos de servicio educativo.

- Reconocimiento profesional entre el personal docente y académico de la entidad (Merriam, 1988).

- Grado académico o estudios de posgrado.

- Experiencia en el tema de asesoría técnico-pedagógica.

Los participantes se seleccionaron a partir de la recomendación y reconocimiento de directivos o autoridades o exautoridades de los diferentes niveles, modalidades y municipios a quienes se les solicitó proporcionaran una lista de los profesores que cumplieran con los criterios de selección.

En total se seleccionaron 42 profesionales de la educación. Se incluyeron profesores frente a grupo, subdirectores, directores, jefes de enseñanza, ATP, supervisores e inspectores de los cinco municipios del estado (Tijuana, Ensenada, Rosarito, Mexicali y Tecate). Se buscó que los participantes trabajaran en los tres niveles de educación básica, preescolar, primaria y secundaria; de los diversos tipos de servicio (escuelas generales, telesecundarias, secundarias técnicas, educación indígena, educación especial y educación física), y de los tres sistemas educativos del estado: federalizado, estatal y municipal (que existe solo en un municipio del estado). Asimismo se incorporaron a los grupos de trabajo un representante de la SEBS y una observadora de la Dirección General de Formación Continua de la SEP. LoS comités de trabajo se integraron cuidando que en cada uno estuvieran representados los actores educativos involucrados en el proceso de asesoramiento de los diferentes niveles, tipos de servicio y municipios.

Los profesores fueron convocados por las autoridades de la SEBS. Esta Secretaría hizo llegar una carta de comisión a los profesores seleccionados y notificó a la autoridad inmediata. Por parte del equipo de investigación de la universidad se envió un correo electrónico a los participantes para proporcionarles información relevante acerca del proyecto y la logística de las sesiones de trabajo.

\section{Preparación de los insumos para la construcción del perfil del ATP}

Se elaboraron los materiales para los participantes. En concreto se redactaron dos documentos (a) el proyecto de investigación en el que se explicaban los objetivos del trabajo y el método de construcción de la propuesta de competencias y (b) el cuadernillo del trabajo en el que se presentaban los dominios y su definición, subdominios, funciones y competencias del perfil del ATP, así como un ejemplo de los parámetros e indicadores, que serviría de guía para los participantes. 


\section{Revisión del perfil propuesto y elaboración de estándares (comité técnico)}

El comité técnico estuvo integrado por doce participantes -con un promedio de 24 años de servicio-, participaban en el sistema educativo en diversas funciones y la mitad de ellos contaba con estudios de posgrado en educación. El objetivo de este comité fue definir los dominios, competencias y parámetros del perfil del ATP a partir de la discusión y el análisis colegiado dirigido por el equipo de investigación. Los integrantes del comité realizaron las siguientes actividades:

- Discutir los dominios y subdominios identificados durante la primera etapa del proyecto de investigación para realizar las adecuaciones pertinentes.

- Definir de manera conjunta los dominios y los subdominios del perfil del ATP.

- Revisar las competencias propuestas y su congruencia con las funciones.

- Redactar los parámetros en subgrupos de trabajo de acuerdo a los dominios del perfil del ATP.

\section{Revisión de dominios, subdominios y competencias y elaboración de indicadores (comité académico)}

El comité académico estuvo conformado por 20 participantes. Contaban con un promedio de 23 años de experiencia docente y 13 de ellos contaban con estudios de posgrado en educación. Se incluyó en este grupo a 2 profesores recientemente jubilados. Este segundo comité tuvo como objetivo redactar los indicadores de cada uno de los parámetros de las competencias del ATP a partir del trabajo colegiado en subgrupos. Los integrantes del comité académico realizaron las siguientes actividades:

- Revisar la definición de los dominios y los subdominios y las funciones del perfil del ATP acordados por el comité técnico.

- Revisar las competencias y parámetros propuestos por el comité técnico.

- Redactar en subgrupos de trabajo los indicadores de cada uno de los parámetros del perfil del ATP.

Al término de la sesión, el comité discutió cuestiones relacionadas con los siguientes aspectos: la transversalidad entre las funciones, competencias, parámetros e indicadores de cada dominio; la congruencia de las competencias, parámetros e indicadores con las políticas educativas vigentes; la relevancia de los parámetros e indicadores para el desarrollo de cada una de las competencias; la claridad de la redacción de las competencias, parámetros e indicadores y el uso de conceptos significativos para los docentes, acordes al discurso educativo que se maneja en el contexto.

Posteriormente se convocó a un representante de cada uno de los subgrupos de trabajo del comité académico para realizar la validación de contenido de la versión final del perfil. Este subgrupo cuidó que las competencias, parámetros e indicadores fueran representativos de los dominios y subdominios del perfil del ATP.

\section{Elaboración de los materiales para la validación de juicios independientes}

El equipo de investigación elaboró materiales para el trabajo del comité de validación. Se elaboraron tres documentos: (a) el proyecto de investigación en el que se explicaban los objetivos del trabajo y el método de construcción de la propuesta de competencias, (b) un cuadernillo con el perfil completo del ATP, es decir, los dominios, subdominios, competencias, parámetros e indicadores y (b) un cuadernillo de respuestas con las instrucciones, los criterios de validación y los parámetros e indicadores que sería evaluados. 


\section{Validación por juicios independientes}

El comité de validación estuvo integrado por 10 docentes en diversas funciones, con un promedio de 25 años de servicio y 7 de ellos con posgrado en educación. El objetivo de este comité fue validar los parámetros e indicadores desarrollados por los comités anteriores mediante la metodología de jueces independientes. Las actividades de sus integrantes fueron las siguientes:

Revisar la definición de los dominios y subdominios del perfil del ATP acordados por el comité académico.

Participar en el proceso de validación de los parámetros e indicadores del perfil del ATP a partir de los criterios de congruencia, relevancia, claridad y suficiencia.

A diferencia de las sesiones anteriores en las que se realizaron grupos de trabajo, en esta etapa se buscó el juicio independiente de los participantes a través de una escala de Likert de cuatro criterios: congruencia, relevancia, claridad y suficiencia.

En la tabla 1 se presentan los criterios, su definición y la escala. Cabe señalar que también se solicitó a los participantes realizar observaciones y sugerencias a los parámetros o indicadores.

Tabla 1. Criterios para validar el perfil del ATP

\begin{tabular}{|c|c|c|}
\hline Criterio & Definición & Escala \\
\hline Congruencia & $\begin{array}{l}\text { Es el grado en que el indicador es consecuente con } \\
\text { el estándar correspondiente. }\end{array}$ & $\begin{array}{l}\text { (5) Muy congruente } \\
\text { (4) Congruente } \\
\text { (3) Indiferente } \\
\text { (2) Poco congruente } \\
\text { (1) No congruente }\end{array}$ \\
\hline Relevancia & $\begin{array}{l}\text { Es el grado en que la información solicitada es } \\
\text { importante para el desarrollo de la práctica asesora. } \\
\text { Su juicio nos ayudará más adelante para diferenciar } \\
\text { niveles de desempeño desde un nivel inicial hasta } \\
\text { uno de experto en el campo de la asesoría técnico- } \\
\text { pedagógica. }\end{array}$ & $\begin{array}{l}\text { (5) Muy relevante } \\
\text { (4) Relevante } \\
\text { (3) Indiferente } \\
\text { (2) Poco relevante } \\
\text { (1) No relevante }\end{array}$ \\
\hline Claridad & $\begin{array}{l}\text { Es el grado en que el indicador comunica de manera } \\
\text { objetiva el enunciado, es decir que no admite más } \\
\text { de una interpretación posible. }\end{array}$ & $\begin{array}{l}\text { (5) Muy claro } \\
\text { (4) Claro } \\
\text { (3) Indiferente } \\
\text { (2) Poco claro } \\
\text { (1) No claro }\end{array}$ \\
\hline $\begin{array}{l}\text { Suficiencia de } \\
\text { los indicadores } \\
\text { del estándar }\end{array}$ & $\begin{array}{l}\text { Es el grado en que se incluyen los indicadores } \\
\text { necesarios para describir cada estándar. }\end{array}$ & $\begin{array}{l}\text { (5) Muy suficiente } \\
\text { (4) Suficiente } \\
\text { (3) Indiferente } \\
\text { (2) Poco suficiente } \\
\text { (1) No suficiente }\end{array}$ \\
\hline
\end{tabular}




\subsection{Análisis de resultados de la validación de juicios independientes}

Para Escurra (1988) uno de los problemas más importantes del método de validación por juicios independientes es el análisis de los resultados. En este caso se utilizó el Coeficiente de variación (Cv) (Berenson y Levine, 1996). Este coeficiente indica el porcentaje de homogeneidad de los juicios de los expertos (Berenson y Levine, 1996; Leyva, 2004). Se calcula a partir de la siguiente fórmula (Berenson y Levine, 1996; Leyva, 2004):

Donde:

$$
\mathrm{Cv}=\frac{\sigma}{\mu} * 100
$$

$\sigma=$ Desviación estándar

$\mu=$ Media

En la tabla 2 se presenta el significado del valor del coeficiente.

Tabla 2. Valores de concordancia entre jueces

\begin{tabular}{|c|c|}
\hline Valores de Cv & Interpretación \\
\hline 0 a $31 \%$ & Juicios homogéneos \\
\hline 32 a $35 \%$ & Distribución normal \\
\hline Mayor a $35 \%$ & Juicios heterogéneos \\
\hline
\end{tabular}

Los diez jueces evaluaron 574 criterios, de los cuales 572 resultaron con un Coeficiente de variación (Cv) menor a 31\%, que significa que los juicios de los participantes fueron homogéneos. Es decir, hubo acuerdo en 572 casos. Solo dos de los criterios presentaron un Cv igual a 34.32\%, que significa que las respuestas de los jueces tuvieron una distribución normal. Los parámetros e indicadores correspondientes fueron identificados y revisados a la luz de los comentarios de los participantes.

En respuestas que presentaron un alto grado de acuerdo se hicieron sugerencias para mejorar la claridad de los enunciados. Los comentarios estuvieron orientados a modificar verbos, mejorar la claridad de los conceptos, complementar la redacción de parámetros e indicadores, señalar similitudes, sintetizar y sugerir indicadores, hacer cuestionamientos y enfatizar la relevancia de los parámetros e indicadores.

\section{Perfil del personal docente con funciones de asesoría técnico pedagógica}

\subsection{Dominios del perfil referencial del ATP}

El dominio (A) "Responsabilidades profesionales", representa los conocimientos y habilidades básicos que se requieren para el desempeño de la función, mientras que los dominios (B) "Asesoría y acompañamiento", (C) "Formación continua de docentes y directivos" y (D) "Evaluación educativa" hacen referencia a los ámbitos específicos de actuación del ATP. En el gráfico 2 se presenta el modelo del perfil. 


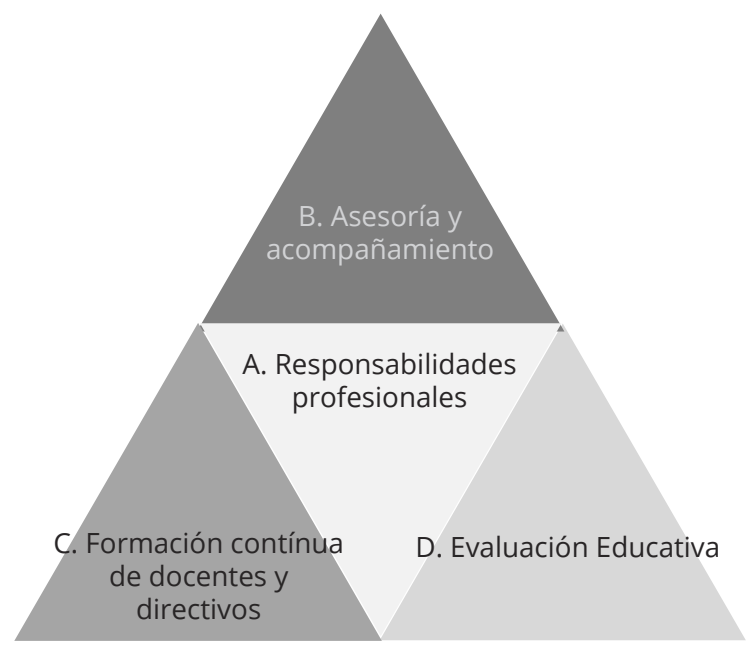

\section{Gráfico 2. Dominios del perfil del ATP}

\subsection{Definición de los dominios del perfil referencial del ATP}

La definición de cada uno de los dominios se presenta en el gráfico 3.

\section{A. Responsabilidades profesionales}

- Conocimientos y habilidades generales para el desempeño de la función de asesoría técnico-pedagógica y procesos de formación individuales y colectivos para la adquisición de los rasgos deseables del perfil del ATP.

\section{B. Asesoría y acompañamiento}

- Apoyo externo que se brinda en los centros escolares de acuerdo con su contexto para la mejora continua. Se relaciona con los procesos de ayuda sostenida dirigida a un docente, directivo o colectivo escolar para la mejora del logro educativo asociados a la gestión escolar, los aprendizajes y prácticas educativas.

\section{Formación continua de docentes y directivos}

- Participación del ATP en el desarrollo de programas y procesos de formación continua adecuados a las necesidades del contexto de los directivos y maestros, que implican la impartición de cursos o talleres.

\section{Evaluación educativa}

- Desarrollo de procesos de evaluación formativa para: identificar las necesidades de asesoría de las escuelas, los docentes y directivos; recolectar y sistematizar información para valorar el logro de los objetivos propuestos; dar seguimiento a los procesos de asesoría y acompañamiento a las escuelas, y; autoevaluar su desempeño como asesor y reflexionar sobre su práctica.

\section{Gráfico 3. Definición de los dominios del perfil del ATP}




\subsection{Los subdominios del perfil del ATP}

Al interior de cada dominio se identificaron subdominios. Se buscó que la organización de los mismos siguiera una lógica interna que diera claridad a la propuesta.

El dominio (A) "Responsabilidades profesionales" se organizó a partir de las temáticas de las competencias identificadas. El dominio (B) "Asesoría y acompañamiento" se estructuró a partir de la lógica propia de un proceso de asesoramiento (Domingo, 2012). El dominio (C) "Formación continua de docentes y directivos" se organizó a partir de las acciones relacionada s con la formación continua en las que puede participar el ATP. El dominio (D) "Evaluación educativa" se planteó a partir de los ámbitos de evaluación en los que se espera que se desempeñe el ATP de acuerdo con la Ley del Instituto Nacional para la Evaluación de la Educación (DOF, 2013b) (véase gráfico 4).

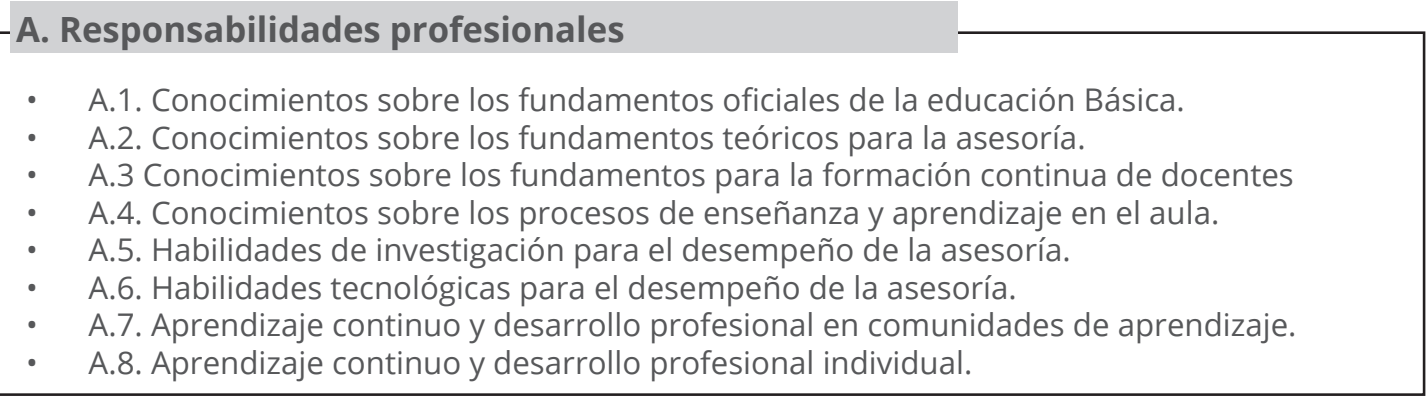

\section{B. Asesoría y acompañamiento}

- B.1. Construye relaciones productivas de trabajo.

- B.2. Identifica necesidades de asesoramiento.

- B.3. Diseña estrategias de asesoría y acompañamiento.

- B.4. Desarrolla acciones de asesoría y acompañamiento.

- B.5. Brinda seguimiento a las acciones de asesoría y acompañamiento.

\section{Formación continua de docentes y directivos}

- C.1. Diseña acciones y materiales para la formación continua de docentes y directivos.

- C.2. Adapta programas para la formación continua de docentes y directivos.

- C.3. Imparte programas de formación continua a docentes y directivos.

\section{Evaluación educativa}

- D.1 .Participa en los procesos de evaluación promovidos por el sistema educativo.

- D.2. Participa en los procesos de evaluación formativa de las escuelas.

- D.3. Evalúa su desempeño como asesor.

\section{Gráfico 4. Subdominios del perfil referencial del ATP}




\subsection{Competencias, parámetros e indicadores del perfil del ATP}

A modo de ejemplo, a continuación se presentan las competencias, parámetros e indicadores de dos subdominios del dominio de asesoría y acompañamiento.

\begin{tabular}{|c|c|c|}
\hline \multicolumn{3}{|c|}{ Dominio B. y acompañamiento } \\
\hline \multicolumn{3}{|c|}{ Subdominio B.1 Construcción de relaciones productivas de trabajo } \\
\hline Competencia & Parámetros & Indicadores \\
\hline $\begin{array}{l}\text { Un ATP que } \\
\text { construye relaciones } \\
\text { productivas de } \\
\text { trabajo: }\end{array}$ & \multirow{6}{*}{$\begin{array}{l}\text { B.1.1 Genera relaciones } \\
\text { de empatía y respeto en } \\
\text { las comunidades escolares } \\
\text { mediante la comunicación } \\
\text { efectiva. }\end{array}$} & $\begin{array}{l}\text { B.1.1.1 Identifica estrategias y técnicas } \\
\text { para una comunicación efectiva y } \\
\text { asertiva. }\end{array}$ \\
\hline \multirow{14}{*}{$\begin{array}{l}\text { Establece relaciones } \\
\text { de empatía y de } \\
\text { respeto con los } \\
\text { diversos actores que } \\
\text { integran la comunidad } \\
\text { educativa mediante la } \\
\text { comunicación efectiva, } \\
\text { la promoción de una } \\
\text { cultura de colaboración } \\
\text { y el desarrollo de } \\
\text { comunidades de } \\
\text { aprendizaje a partir } \\
\text { del conocimiento sobre } \\
\text { manejo de grupos, el } \\
\text { logro de consensos, } \\
\text { la prevención } \\
\text { de conflictos y } \\
\text { la resolución } \\
\text { de situaciones } \\
\text { problemáticas. }\end{array}$} & & $\begin{array}{l}\text { B.1.1.2 Utiliza estrategias de } \\
\text { comunicación efectiva en los contextos } \\
\text { escolares. }\end{array}$ \\
\hline & & $\begin{array}{l}\text { B.1.1.3 Manifiesta una actitud tolerante } \\
\text { y respetuosa en la comunidad educativa. }\end{array}$ \\
\hline & & $\begin{array}{l}\text { B.1.1.4 Escucha con atención y de } \\
\text { manera inclusiva las opiniones de todos } \\
\text { los asesorados. }\end{array}$ \\
\hline & & $\begin{array}{l}\text { B.1.1.5 Manifiesta una actitud de } \\
\text { empatía ante las características y } \\
\text { problemáticas de los colectivos. }\end{array}$ \\
\hline & & $\begin{array}{l}\text { B.1.1.6 Reconoce las características de } \\
\text { los interlocutores y muestra empatía } \\
\text { y respeto a las problemáticas que los } \\
\text { afectan. }\end{array}$ \\
\hline & \multirow{4}{*}{$\begin{array}{l}\text { B.1.2 I mpulsa el desarrollo } \\
\text { de comunidades de } \\
\text { aprendizaje en su ámbito } \\
\text { de influencia. }\end{array}$} & $\begin{array}{l}\text { B.1.2.1 Describe las características de } \\
\text { las comunidades de aprendizaje. }\end{array}$ \\
\hline & & $\begin{array}{l}\text { B.1.2.2 Explica estrategias para lograr } \\
\text { el aprendizaje entre pares a través de } \\
\text { comunidades de aprendizaje. }\end{array}$ \\
\hline & & $\begin{array}{l}\text { B.1.2.3 Identifica condiciones } \\
\text { viables para formar comunidades de } \\
\text { aprendizaje. }\end{array}$ \\
\hline & & $\begin{array}{l}\text { B.1.2.4 Aplica, da seguimiento y evalúa } \\
\text { resultados en conjunto con los actores } \\
\text { involucrados en la asesoría. }\end{array}$ \\
\hline & \multirow{3}{*}{$\begin{array}{l}\text { B.1.3 Promueve el trabajo } \\
\text { colaborativo con los } \\
\text { diferentes agentes en } \\
\text { su ámbito de influencia } \\
\text { mediante su participación } \\
\text { activa en los grupos de } \\
\text { trabajo. }\end{array}$} & $\begin{array}{l}\text { B.1.3.1 Reconoce las diferencias } \\
\text { profesionales de los integrantes del } \\
\text { colectivo. }\end{array}$ \\
\hline & & $\begin{array}{l}\text { B.1.3.2 Caracteriza los principios del } \\
\text { trabajo colaborativo en el ámbito } \\
\text { educativo. }\end{array}$ \\
\hline & & $\begin{array}{l}\text { B.1.3.3 Incluye a todos los integrantes } \\
\text { del colectivo docente en las reuniones de } \\
\text { trabajo colegiado. }\end{array}$ \\
\hline & \multirow{2}{*}{$\begin{array}{l}\text { B.1.4 Previene posibles } \\
\text { conflictos de intereses } \\
\text { entre los docentes, los } \\
\text { directivos y otros agentes } \\
\text { educativos. }\end{array}$} & $\begin{array}{l}\text { B.1.4.1 Reconoce los riesgos que pueden } \\
\text { afectar el buen clima de trabajo en su } \\
\text { ámbito de influencia. }\end{array}$ \\
\hline & & $\begin{array}{l}\text { B.1.4.2 Sugiere técnicas para el manejo } \\
\text { de conflictos de intereses entre los } \\
\text { integrantes del colectivo de su ámbito de } \\
\text { influencia. }\end{array}$ \\
\hline
\end{tabular}




\begin{tabular}{|c|c|c|}
\hline \multicolumn{3}{|c|}{ Dominio B. Asesoría y acompañamiento } \\
\hline \multicolumn{3}{|c|}{ Subdominio B. 2 Identifica necesidades de asesoramiento } \\
\hline Competencia & Parámetros & Indicadores \\
\hline \multirow{10}{*}{$\begin{array}{l}\text { Un ATP que identifica las } \\
\text { necesidades de asesoría: } \\
\text { Comprende el contexto } \\
\text { social, económico, cultural } \\
\text { y educativo en el que } \\
\text { se desarrolla la práctica } \\
\text { (zona, escuela o aula) para } \\
\text { identificar las necesidades } \\
\text { de asesoramiento de } \\
\text { los docentes, escuelas } \\
\text { y directivos, a partir de } \\
\text { procesos sistemáticos en los } \\
\text { que se tome en cuenta la } \\
\text { opinión de los implicados. }\end{array}$} & \multirow{5}{*}{$\begin{array}{l}\text { B.2.1 Realiza } \\
\text { diagnósticos de } \\
\text { necesidades de } \\
\text { asesoramiento } \\
\text { en su ámbito de } \\
\text { influencia. }\end{array}$} & $\begin{array}{l}\text { B.2.1.1 Identifica la metodología para } \\
\text { hacer diagnósticos y detectar necesidades } \\
\text { de asesoramiento en su ámbito de } \\
\text { influencia. }\end{array}$ \\
\hline & & $\begin{array}{l}\text { B.2.1.2 Identifica tipos de diagnóstico y } \\
\text { su metodología para detectar necesidades } \\
\text { de asesoramiento. }\end{array}$ \\
\hline & & $\begin{array}{l}\text { B.2.1.3 Argumenta la selección de } \\
\text { estrategias para la detección de } \\
\text { necesidades de asesoramiento. }\end{array}$ \\
\hline & & $\begin{array}{l}\text { B.2.1.4 Adapta instrumentos para } \\
\text { el diagnóstico de necesidades de } \\
\text { asesoramiento de acuerdo a los objetivos } \\
\text { planteados. }\end{array}$ \\
\hline & & $\begin{array}{l}\text { B.2.1.5 Aplica instrumentos de } \\
\text { diagnóstico para detectar necesidades de } \\
\text { asesoramiento en su ámbito de influencia. }\end{array}$ \\
\hline & \multirow{5}{*}{$\begin{array}{l}\text { B.2.2 Analiza } \\
\text { la información } \\
\text { generada a partir } \\
\text { del diagnóstico de } \\
\text { necesidades de } \\
\text { asesoramiento. }\end{array}$} & $\begin{array}{l}\text { B.2.2.1 Sistematiza la información de los } \\
\text { diagnósticos de necesidades. }\end{array}$ \\
\hline & & $\begin{array}{l}\text { B.2.2.2 Integra información útil para el } \\
\text { diagnóstico de necesidades. }\end{array}$ \\
\hline & & $\begin{array}{l}\text { B.2.2.3 Explica al colectivo las } \\
\text { necesidades de asesoramiento } \\
\text { detectadas. }\end{array}$ \\
\hline & & $\begin{array}{l}\text { B.2.2.4 Prioriza las necesidades de } \\
\text { asesoría en conjunto con el colectivo } \\
\text { escolar. }\end{array}$ \\
\hline & & $\begin{array}{l}\text { B.2.2.5 Sugiere ámbitos de mejora para } \\
\text { atender las necesidades de asesoramiento } \\
\text { identificadas. }\end{array}$ \\
\hline
\end{tabular}

\section{Consideraciones finales sobre el proceso de elaboración del perfil}

La reforma educativa mexicana instrumentada a partir de la reforma constitucional y de la Ley General de Educación y la promulgación de dos leyes secundarias regula la carrera docente en nuestro país. Previo a esta reforma, ciertamente existían diversos instrumentos que normaban el ingreso, la promoción en la función vía escalafón vertical y una compensación salarial vía escalafón horizontal (denominada Carrera Magisterial). Lo novedoso de esta reforma es que integra todos estas etapas en un único instrumento jurídico de observancia nacional, añade la regulación de la permanencia en el servicio vía evaluación del desempeño y excluye a los sindicatos de la regulación de la carrera docente.

Otra de las novedades de esta reforma es el reconocimiento de la figura del asesor técnicopedagógico en el sistema educativo nacional. A partir de esta reforma, en concreto la LGSPD, se pretende regular el ingreso y permanencia en la función de asesoría, y enfocar las funciones del asesor a tareas del ámbito pedagógico. En este contexto normativo, el asesor se define como un agente de mejora de la calidad educativa con funciones estrictamente pedagógicas (DOF, 2013d).

La asesoría o apoyo técnico-pedagógico a la escuela es una práctica conocida en otros sistemas educativos iberoamericanos, como por ejemplo España (Domingo, 2012), Argentina (Aguerrondo y Vezub, 2011), Chile (Chile, Ministerio de Educación, 2012) y Perú 
(Perú, Ministerio de Educación, 2014). Para México la definición de la función asesora representó un reto en tanto que se trataba de ordenar una figura ya existente pero que no estaba asociada a actividades pedagógicas. No obstante, se reconoce que varias entidades federativas ya habían avanzado en el sentido de asignar funciones pedagógicas a los maestros comisionados a la función de asesor pedagógico (SEP y OEI, 2006). Sin embargo, difícilmente podían avanzar las entidades federativas en la regulación del ATP, dado que se carecía de una normativa federal que reconociera esta figura en la estructura laboral.

La reforma educativa resolvió en el marco de la LGSPD la indefinición laboral de este actor educativo. La figura está definida pero aún queda pendiente clarificar las instancias administrativas desde las cuales operarán los ATP, es decir cuál será su adscripción institucional. Por esta razón, el perfil se elaboró desde una perspectiva integral y general de las funciones que debe realizar el asesor, independientemente de si se les ubica en escuela, zona, sector de educación básica, centro de maestros, o si se encuentra adscrito a algún nivel o modalidad educativo o bien como apoyo a un programa educativo.

En cuanto a la metodología para la construcción del perfil del ATP, se cuidó que se tomaran en cuenta los documentos oficiales del sistema educativo que han orientado la función, los planteamientos realizados en las leyes secundarias publicadas a partir de la Reforma Educativa y la literatura nacional e internacional en la materia. Se atendieron las recomendaciones de organismos internacionales en el sentido de que los estándares deben ser consensuados y validados por los propios profesionales de la educación, por lo que una condición fundamental en el proceso de elaboración del perfil fue la participación de los profesores de los cinco municipios de Baja California, seleccionados por su compromiso profesional, el reconocimiento de sus pares y su nivel académico. Se incluyó tanto a los usuarios del servicio de apoyo externo como a los propios agentes que prestan este tipo de servicio, asesores y supervisores. En este sentido, se cuenta con la garantía de que el perfil puede ser presentado y discutido con grupos profesionales más amplios por el reconocimiento a la trayectoria de los compañeros que participaron en el proyecto.

El perfil referencial identifica cuatro dominios: (A) Responsabilidades profesionales, (B) Asesoría y acompañamiento, (C) Formación continua de docentes y directivos, y (D) Evaluación educativa. El primer domino responde a los saberes y habilidades básicos para el desempeño de la función asesora, mientras que los otros tres se refieren a los ámbitos específicos de trabajo del ATP. Cada uno de estos ámbitos de trabajo puede ampliarse o eliminarse en función de las decisiones que tome la entidad federativa o la federación en su proceso de regulación de esta figura, por lo que el perfil es flexible.

El desempeño de cada ámbito de trabajo del perfil del ATP podría requerir de un profesional especializado. Sin embargo, los comités decidieron que el perfil se conformara por los cuatro dominios antes mencionados a fin de que reflejara la complejidad de la tarea del asesor. En los grupos de trabajo se discutieron los alcances y limitaciones de la función de asesoría y su problemática, la que se espera que un perfil de estas características ayude a regularizar.

Es importante señalar que el perfil resultado de esta investigación es altamente sensible al contexto educativo, ya que los dominios, subdominios, parámetros e indicadores pueden variar en función de la estructura del sistema educativo de cada entidad federativa. De ahí que se considera que el contexto también determinará los alcances de la función asesora. En este sentido, para que el personal docente en funciones de ATP pueda desempeñarse 
con base en este perfil es necesario garantizar que la propia estructura favorezca las condiciones para el desempeño de la función.

Tal como lo señala la LGSPD, esta propuesta de perfil se entregó a las autoridades de la SEP para su consideración y como aportación para la construcción de la versión nacional oficial. Además de constituir una aportación validada y fundamentada para la federación, este perfil puede ser usado localmente para la definición de programas de formación y para la definición de políticas locales.

\section{Referencias bibliográficas}

Aguerrondo, I. y Vezub, L. (2011). Asesoramiento y desarrollo profesional de los docentes: procesos clave del liderazgo efectivo para la mejora de la escuela. Recuperado de http:// www.uca.edu.ar/uca/common/grupo82/files/Aguerrondo-Vezub-Handbook-BA-espanolAsesoramiento-y-desarrollo-profesional-de-los-docentes.pdf

Arnaut, A. (2006). La función de apoyo técnico-pedagógico. Su relación con la supervisión y la formación continua. En SEP-OEI (Eds.), La asesoría a las escuelas. Reflexiones para la mejora educativa y la formación continua de los maestros (pp. 15-27). México, SEP-OEI.

Barrera, I., y Myers, R. (2011). Estándares y evaluación docente en México: el estado del debate. Santiago, Preal.

Berenson, M., y Levine, D. (1996). Estadística básica en administración. Conceptos y aplicaciones (6.a ed.). México, Prentice Hall.

Bonilla, O. (2006). La asesoría técnica a la escuela. En SEP-OEI (Eds.). La asesoría a las escuelas. Reflexiones para la mejora educativa y la formación continua de los maestros (pp. 29-49). México, SEP-OEI.

Calvo, B. (2007). Los asesores técnico-pedagógicos y el fortalecimiento de las escuelas de educación básica. Presentado en el IX Congreso Nacional de Investigación Educativa. Yucatán, México, Comie-UADY.

Cano, E. (2012). Conceptualización y evaluación de competencias docentes. En Cisneros, E., García, B., Luna, E. y Marín, R. (Coords.), Evaluación de competencias docentes en educación superior (pp. 309-338). México, ReDECA-Juan Pablo Editor.

Celis, M. (2012). Diagnóstico y desarrollo de competencias docentes en el sistema escolar. Resultados de una experiencia preliminar con base en el modelo de gestión escolar de fundación Chile. En Cisneros, E., García, B., Luna, E. y Marín, R. (Coords.), Evaluación de competencias docentes en educación superior. (pp.339-376). México, ReDECA-Juan Pablo Editor.

Chile, Gobierno de Chile (2011). Marco para la buena enseñanza. Autor.

Chile, Ministerio de Educación (2012). Modelo para el apoyo técnico-pedagógico a los establecimientos educacionales del país. Autor.

Cordero, G., Fragoza, A. y Vázquez, M. A. (2015). El servicio de asistencia técnica a la escuela: aproximaciones a su configuración en México. Revista electrónica de investigación educativa, 17(1), 55-71. Recuperado de http://redie.uabc.mx/vol17no1/contenido-cordero-fragozav. $\underline{\mathrm{html}}$ 
Danielson, C. (2013). The framework. The Danielson Group. Recuperado de http:// danielsongroup.org/framework/

Diario Oficial de la Federación [DOF] (2013a). Plan Nacional de Desarrollo 2013-2018. Publicado en el Diario Oficial de la Federación el 20 de mayo de 2013. Recuperado de: www.dof.gob.mx/nota_detalle.php?\%20codigo=5299465\&fecha=20/05/2013.

DOF (2013b). Ley del Instituto Nacional para la Evaluación de la Educación. Publicado en el Diario Oficial de la Federación el 11 de setiembre de 2013. Recuperado de: http://www.sep. gob.mx/work/appsite/reforma_educativa/Ley_del_Instituto_Nacional_para_la_Evaluacioon de la Educacion.pdf

DOF (2013c). Ley general de educación. Publicado en el diario oficial de la Federación el 11 de setiembre de 2013. Recuperado de: https://www.sep.gob.mx/work/models/sep1/ Resource/3f9a47cc-efd9-4724-83e4-0bb4884af388/ley_general_educacion.pdf

DOF (2013d). Ley general del servicio profesional docente. Publicado en el Diario Oficial de la Federación el 11 de setiembre de 2013. Recuperado de: http://www.diputados.gob.mx/ LeyesBiblio/pdf/LGSPD.pdf

DOF (2013e). Acuerdo número 712 por el que se emiten las reglas de operación del programa para el desarrollo profesional docente. Publicado en el Diario Oficial de la Federación el 29 de diciembre de 2013. Recuperado de: http://dof.gob.mx/nota_detalle.php?codigo=5328 399\&fecha=29/12/2013.

Domingo, J. (2012). Funciones de asesoramiento. En J. Domingo (Ed.), Asesoramiento al Centro Educativo. Colaboración y Cambio en la Institución (3.a ed., pp. 183-202). Barcelona, Octaedro.

Elo, S., y Kyngäs, H. (2008). The qualitative content analysis process. Journal of Advanced Nursing, 62(1), 107-115.

Escurra, L. (1988). Cuantificación de la validez de contenido por criterio de jueces. Revista de psicología de la PUCP, 6, 1-2, 103-111. Recuperado de http://revistas.pucp.edu.pe/index. php/psicologia/article/view/4555/4534

Ingvarson, L., y Kleinhenz, E. (2006). Estándares profesionales de práctica y su importancia para la enseñanza. Revista de educación, (340), 265-298.

Leyva, Y. (2004). Evaluación de competencias médicas: validez de constructo de pruebas criteriales (Tesis doctoral). México, Universidad Autónoma de Aguascalientes.

Merriam, S. (1988). Case study research in education: a qualitative approach. San Francisco, Jossey-Bass.

Meza, M., Vázquez, M. y Cordero, G. (2008). Las funciones del asesor técnico-pedagógico. Análisis de la documentación oficial. Presentado en I Congreso Latinoamericano de Ciencias de la Educación. Baja California, México, UABC.

Orealc/Unesco. (2013). Antecedentes y criterios para la elaboración de políticas docentes en América Latina y el Caribe. Santiago, Autor. 
Ornelas, C. (2013). Si se da marcha atrás en reforma educativa, Peña Nieto se acaba. Periódico Siempre! Publicado el 21 de setiembre de 2013. Recuperado el 11 de febrero de 2013 de: http://www.siempre.com.mx/2013/09/si-se-da-marcha-atras-en-reformaeducativa-pena-nieto-se-acaba/

Perú, Ministerio de Educación de Perú. (2012). Marco de buen desempeño docente. Autor.

Perú, Ministerio de Educación (2014). Protocolo de acompañamiento pedagógico. Autor. Recuperado de: http://www2.minedu.gob.pe/digesutp/formaciondeformadores/wpdescargas/2014/01_Protocolos_acompamiento.pdf

Sánchez, R., Cordero, G. y Bocanegra, N. (2009). El asesor de zona escolar de educación primaria en Tijuana. Percepciones y contradicciones en su función. Presentado en el X Congreso Nacional de Investigación Educativa. Veracruz, México, Comie-Universidad Veracruzana.

Schmelkes, S. (2014). Evaluación del desempeño docente: estado de la cuestión. Conferencia magistral impartida en el V Coloquio de la Red Iberoamericana de Investigadores sobre Evaluación de la Docencia. Ensenada, Baja California.

Secretaría de Educación Pública [SEP] (2005). Orientaciones generales para constituir y operar el servicio de asesoría académica a la escuela. México, Autor.

SEP (2010). Estándares de desempeño docente en el aula para la educación básica en México. México, Autor.

SEP (2012). Colección criterios para el fortalecimiento del sistema regional de asesoría académica a la escuela. México, Autor.

SEP (2014). Perfil, parámetros e indicadores para docentes y técnicos docentes. México, Autor. Recuperado de: http://servicioprofesionaldocente.sep.gob.mx/content/ba/docs/ parametros indicadores/Completo.pdf

SEP y OEI (2006). Historias de aprendizaje sobre la asesoría y la formación continua en México. México: Autor.

SEBS (2013). Lineamientos normativos para la gestión institucional, escolar y pedagógica para el ciclo escolar 2013-2014. México, Autor.

Stronge, J., y Tucker, P. (2003). Handbook on Educational Specialist Evaluation. Assessing and Improving Performance. Estados Unidos: Eye on Education.

Tapia, G. (2008). Formación para el asesoramiento a las escuelas: un proceso emergente en México. Profesorado: Revista de curriculum y formación del profesorado, 12(1), 1-15.

Vaillant, D. (2004). Construcción de la profesión docente en América Latina. Tendencias, temas y debates. Preal. Recuperado de: http://www.oei.es/docentes/articulos/construccion profesion_docente_AL_vaillant.pdf 
Vázquez, M., Meza, M. y Cordero, G. (2012). La oferta formativa dirigida a asesores técnicopedagógicos en el catálogo nacional de formación continua y superación profesional para maestros de educación básica en servicio. Presentado en el Primer Congreso Nacional de Investigación y Desarrollo en Educación. Yucatán, México, UADY.

Vázquez, M., Meza, M., Cordero, G. y Patiño, X. (2012). La oferta formativa aprobada para la función de asesoría. Análisis de los catálogos nacionales (2008-2012). Diálogos sobre educación, 3(6), 1-14.

Vázquez, M.A., Cordero, G. y Y. Leyva. (2014). Análisis comparativo de los criterios de desempeño profesional para la enseñanza en cuatro países de América. Actualidades Investigativas en Educación, 14 (3), 1-20. Recuperado de: http://www.redalyc.org/ pdf/447/44732048018.pdf

Zebadúa, E. (2013). Reforma educativa en México. La crónica de hoy. Publicado el 10 de febrero de 2013. Recuperado el 11 de febrero de 2013 de: http://www.cronica.com.mx/ notas/2011/556315.html

\section{(Endnotes)}

${ }^{1}$ Esta propuesta se desarrolló de junio de 2013 a abril de 2014 gracias al financiamiento del Consejo Nacional de Ciencia y Tecnología (Conacyt) (Convocatoria SEP-Conacyt 2010 Proyecto No. 160836) y el apoyo y facilidades otorgados por la Secretaría de Educación y Bienestar Social (SEBS) del Gobierno del Estado de Baja California, México.

${ }^{2}$ El concepto de parámetros sustituye al de estándares en tanto que este último término fue ampliamente cuestionado en el proceso de definición de la LGSPD. Al ser la docencia una práctica que en México se desempeña en contextos sociales y económicos extremos, el término se confundió con estandarización, que se refiere a que una situación se desarrolla bajo las mismas condiciones (Schmelkes, 2014) y fue rechazado por los sindicatos de maestros. 